\title{
Aspectos dermatoscópicos na psoríase ungueal
}

\author{
Dermoscopy in nail psoriasis
}

\author{
Débora Cadore de Farias ${ }^{1}$ \\ Nilton Di Chiacchio ${ }^{3}$
}

\author{
Antonella Tosti ${ }^{2}$ \\ Sergio Henrique Hirata ${ }^{4}$
}

\begin{abstract}
Resumo: Os autores relatam sua experiência no uso da dermatoscopia na psoríase ungueal e descrevem os achados dessa ferramenta diagnóstica.

Palavras-chave: Doenças da unha; Psoríase; Psoríase/diagnóstico
\end{abstract}

\begin{abstract}
The authors report on their experience with the use of dermoscopy in nail psoriasis and describe their findings with this diagnostic tool.

Keywords: Nail diseases; Psoriasis; Psoriasis/diagnosis.
\end{abstract}

\section{INTRODUÇÃO}

A dermatoscopia é um procedimento largamente utilizado para auxiliar o diagnóstico de lesões cutâneas (melanocíticas ou não). ${ }^{1}$ No aparelho ungueal essa ferramenta é consagrada apenas para lesões melanocíticas. ${ }^{2}$ Em lesões não melanocíticas do aparelho ungueal esse exame é recente e de grande valia. Entretanto ainda não há estudos suficientes que validem seu uso e muito menos que substituam a biópsia ungueal.

Os achados dermatoscópicos da psoríase ungueal dependem de qual parte do aparelho ungueal é afetada pela doença. ${ }^{3} \mathrm{Na}$ matriz ungueal a psoríase produz anormalidades na superfície da lâmina ungueal, como o pitting. Quando acomete o leito ungueal, pode produzir onicólise, manchas salmão, hemorragias $\mathrm{e}$ hiperqueratose subungueal. A dermatoscopia permite visualizar melhor essas alterações na lâmina e no leito ungueal, bem como detectar alterações vasculares em hiponíquio e dobra ungueal proximal indicativas da doença.

Para a realização da dermatoscopia da lâmina ungueal na psoríase, é necessário um aumento de, pelo menos, 30 vezes. Podem-se utilizar aparelhos de luz polarizada ou não. Quando utilizado o de luz não polarizada, prefere-se a utilização de um gel transparente como substância de interface, pois ele preenche o espaço entre a superfície convexa da lâmina ungueal e a plana da lente do dermatoscópio. Os achados da dermatoscopia da lâmina ungueal na psoríase ungueal estão descritos no quadro 1 .

Quando há a presença de onicólise, a dermatoscopia geralmente permite a visualização de borda eritematosa, muitas vezes subclínica e não visível a olho nu, representada por mancha vermelhoalaranjada circundando a área de onicólise. Esse achado é específico da onicólise da psoríase ungueal (Figura 1A).

As hemorragias em estilhaço são resultado do sangramento nos capilares do leito ungueal dispostos de maneira longitudinal e sucessiva incorporação do sangue na lâmina ungueal ventral. Esse achado não é específico da psoríase. São frequentemente observadas também em onicomicoses, dermatite de contato e traumas no aparelho ungueal (Figura 1A).

Para a realização da dermatoscopia do hiponíquio, podem-se também utilizar aparelhos de luz

Recebido em 02.07.2009.

Aprovado pelo Conselho Consultivo e aceito para publicação em 18.12.09.

Trabalho realizado na clínica de dermatologia - Hospital do Servidor Público do Estado de São Paulo - São Paulo (SP), Brasil.

Conflito de interesse: Nenhum / Conflict of interest: None

Suporte financeiro: Nenhum / Financial funding: None

Médica dermatologista. Clínica de dermatologia - Santa Casa de São Paulo - São Paulo (SP), Brasil

Professora catedrática de dermatologia da Universidade de Bolonha - Bolonha, Itália

Médico assistente. Clínica de dermatologia - Hospital do Servidor Público do Estado de São Paulo - São Paulo (SP), Brasil

Doutor em medicina pela Universidade Federal de São Paulo (Unifesp), médico do departamento de dermatologia da Universidade Federal de São Paulo (Unifesp), ABC - São Paulo (SP), Brasil. 
QuADro 1: Descrição dos achados dermatoscópicos de lâmina ungueal na psoríase ungueal

\begin{tabular}{|ll|}
\hline Achado & Descrição dermatoscópica \\
Pitting & $\begin{array}{l}\text { Depressões irregulares em formato e tamanho, circundadas por um halo esbranquiça } \\
\text { do } \\
\text { Manchas irregulares em tamanho e formato, com coloração variando do vermelho ao } \\
\text { alaranjado } \\
\text { Área homogeneamente esbranquiçada ou composta de múltiplas estriações longitudi } \\
\text { Onicólise }\end{array}$ \\
$\begin{array}{l}\text { nais, geralmente circundada por mancha vermelho-alaranjada } \\
\text { Memorragias em estilhaço }\end{array}$ & $\begin{array}{l}\text { Vasos dilatados e tortuosos observados no leito ungueal distal } \\
\text { Vasos sanguíneos }\end{array}$ \\
\hline
\end{tabular}
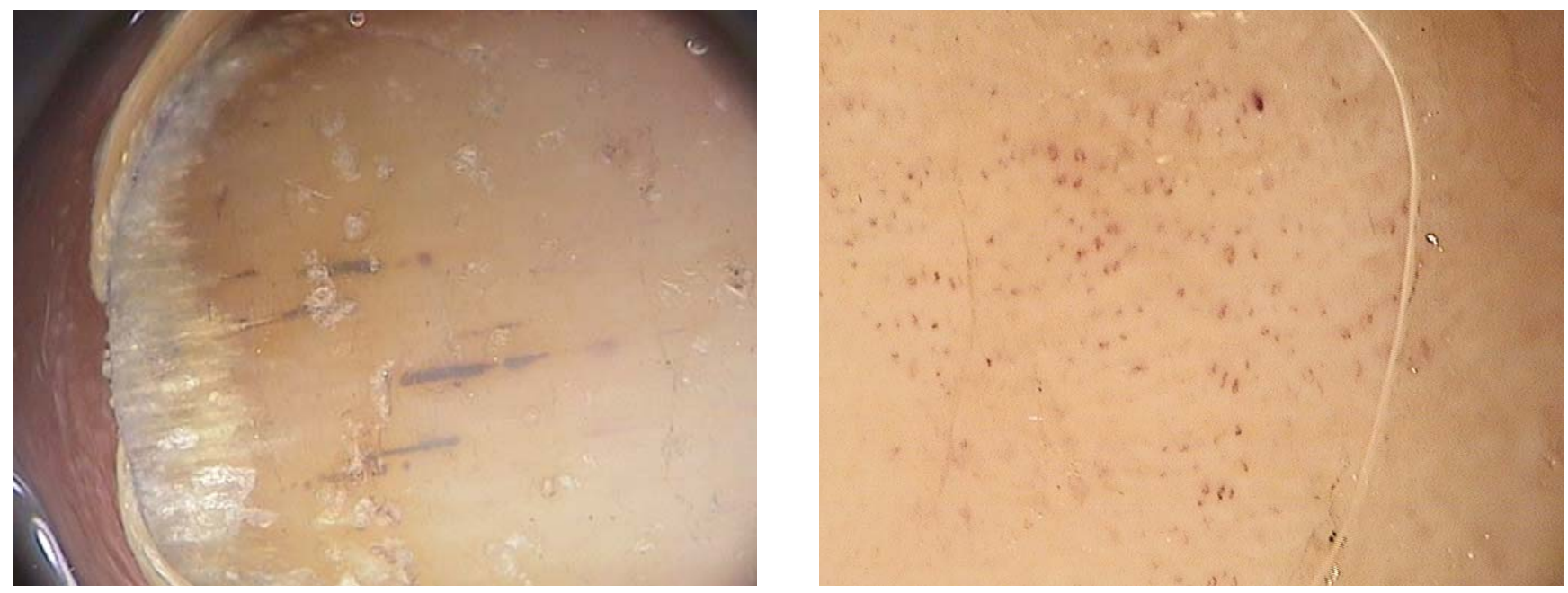

Figura 1: A. Dermatoscopia da lâmina ungueal. Nota-se a onicólise caracterizada por uma área esbranquiçada composta de várias finas estriações longitudinais. O pitting aparece como depressões irregulares em tamanho e formato, circundadas por uma borda esbranquiçada e preenchidas pelo gel. Observam-se também hemorragias em estilhaço, caracterizadas por estriações longitudinais purpúricas;

B. Dermatoscopia do hiponíquio. Observa-se aumento da densidade capilar, com capilares dilatados, tortuosos e de distribuição irregular

polarizada ou não. Notam-se capilares dilatados, alongados e tortuosos, com distribuição irregular (Figura 1B). É necessário um aumento de, no mínimo, 40 vezes para visualização dos capilares anormais. Iorizzo e colaboradores, em 2008, demonstraram que a densidade capilar é correlacionada positivamente com a gravidade da doença. Esse estudo também demonstrou que a análise quantitativa dos capilares se correlaciona com a resposta ao tratamento. Nesse estudo, os pacientes, após três meses de tratamento tópico com calcipotriol, apresentaram uma redução no número de capilares. ${ }^{4}$

$\mathrm{Na}$ experiência dos autores, a dermatoscopia do hiponíquio é a melhor ferramenta para confirmação do diagnóstico de psoríase em pacientes com onicólise isolada ou leve hiperqueratose do leito ungueal. ${ }^{3}$

A dermatoscopia da dobra ungueal proximal é também útil para avaliar a gravidade da doença, encontrando-se alterações quantitativas e morfológicas de capilares. O número e o diâmetro dos capilares encontram-se significativamente diminuídos. ${ }^{5,6,7}$

Zaric e colaboradores, em 1982, encontraram uma diminuição no tamanho dos capilares da dobra ungueal proximal nos pacientes com psoríase cutânea e articular, quando comparados com o grupo controle.

Bhushan e colaboradores, em 2000, observaram uma diminuição na densidade capilar na dobra ungueal proximal em pacientes com psoríase ungueal ou psoríase com acomentimento ungueal e articular quando comparados com o grupo controle. Apenas nos pacientes com psoríase articular associada ou não ao acometimento ungueal foi evidenciada também uma diminuição no diâmetro desses capilares.

Concluindo, a dermatoscopia é um exame não invasivo, de aplicação rápida e baixo custo, que pode auxiliar no diagnóstico de psoríase ungueal em casos duvidosos e melhorar o acompanhamento do paciente em relação à resposta ao tratamento instituído. 


\section{REFERÊNCIAS}

1. Rezze GG, Soares de Sá BC, Neves RI. Dermatoscopia: o método de análise de padrões. An Bras Dermatol. 2006;81:261-8.

2. Tosti A, Piraccini BM, Farias DC. Dealing with melanonychia. Seminars in Cutaneous Medicine and Surgery. 2009;28:49-54.

3. Tosti A, Pirraccini BM, Farias DC. Nail dermoscopy. In: Micali G. Videodermatoscopy in Clinical Pratice. London: Informa Healthcare; 2009.

4. Iorizzo M, Dahdah $M$, Vicenzi C, Tosti A. Videodermoscopy of the hyponychium in nail bed psoriasis. J Am Acad Dermatol. 2008;58:714-5.

5. Ohtsuka T, Yamakage A, Miyachi Y. Statistical definition of nailfold capillary pattern in patients with psoriasis. Int J Dermatol. 1994;33:779-82.
6. Zaric D, Clemmensen OJ, Worm AM, Stahl D. Capillary microscopy of the nail fold in patients with psoriasis and psoriatic arthritis. Dermatologica. 1982;164:10-4.

7. Bhushan M, Moore T, Herrick AL, Griffiths CEM. Nailfold video capillaroscopy in psoriasis. $\mathrm{Br} \mathrm{J}$ Dermatol. 2000;142:1171-6.

\footnotetext{
ENDEREÇO PARA CORRESPONDÊNCIA / MAILING ADDRESS: Débora Cadore de Farias

Rua João Moura, 975 - ap. 164 -Jardim América 05412002 São Paulo, SP

E-mail:decadorefarias@botmail.com
}

Como citar este artigo/How to cite this article: Farias DC, Tosti A, Di Chiacchio N, Hirata SH. Aspectos dermatoscópicos na psoríase ungueal. An Bras Dermatol. 2010;85(1):101-3. 